\title{
ALTERNATIVE ALGEBRAS OVER AN ARBITRARY FIELD
}

\author{
R. D. SCHAFER ${ }^{1}$
}

The results of M. Zorn concerning alternative algebras ${ }^{2}$ are incomplete over modular fields since, in his study of alternative division algebras, Zorn restricted the characteristic of the base field to be not two or three. In this paper we present first a unified treatment of alternative division algebras which, together with Zorn's results, permits us to state that any alternative, but not associative, algebra $A$ over an arbitrary field $F$ is central simple (that is, simple for all scalar extensions) if and only if $A$ is a Cayley-Dickson algebra ${ }^{3}$ over $F$.

A. A. Albert in a recent paper, Non-associative algebras I: Fundamental concepts and isotopy, ${ }^{4}$ in troduced the concept of isotopy for the study of non-associative algebras. We present in the concluding section of this paper theorems concerning isotopes (with unity quantities) of alternative algebras. The reader is referred to Albert's paper, moreover, for definitions and explanations of notations which appear there and which, in the interests of brevity, have been omitted from this paper.

1. Alternative algebras. A distributive algebra $A$ is called an alternative algebra if $a x^{2}=(a x) x$ and $x^{2} a=x(x a)$ for all elements $a$, $x$ in $A$. That is, in terms of the so-called right and left multiplications, $A$ is alternative if $R_{x^{2}}=\left(R_{x}\right)^{2}$ and $L_{x^{2}}=\left(L_{x}\right)^{2}$.

The following lemma, due to R. Moufang, ${ }^{5}$ and the Theorem of Artin are well known.

LEMma 1. The relations $L_{x} R_{x} R_{y}=R_{x y} L_{x}, R_{x} L_{x} L_{y}=L_{y x} R_{x}$, and $R_{x} L_{x y}$ $=L_{y} L_{x} R_{x}$ hold for all $a, x, y$ in an alternative algebra $A$.

Presented to the Society, April 18, 1942; received by the editors December 7, 1942.

1 This paper is the essential portion of the author's doctoral dissertation, written at The University of Chicago under the direction of Professor A. A. Albert, whose kindness the author gratefully acknowledges.

${ }^{2}$ M. Zorn, Theorie der Alternativen Ringe, Abh. Math. Sem. Hamburgischen Univ. vol. 8 (1930) pp. 123-147; Alternativkörper und Quadratische Systeme, ibid. vol. 9 (1933) pp. 395-402. See M. Zorn, Alternative rings and related questions I: Existence of the radical, Ann. of Math. (2) vol. 42 (1941) pp. 676-686, to verify that the Peirce decomposition in Zorn's first paper is valid for $F$ of characteristic two.

${ }^{3}$ Defined over an arbitrary field by A. A. Albert in his Quadratic forms permitting composition, Ann. of Math. (2) vol. 43 (1942) pp. 161-177.

${ }^{4}$ Ann. of Math. (2) vol. 43 (1942) pp. 685-708.

${ }^{5}$ R. Moufang, Zur Struktur von Alternativkörpern, Math. Ann. vol. 110 (1934) pp. 416-430. 
ThEOREM OF ARTin. The subalgebra generated by any two elements of an alternative algebra $A$ is associative.

It follows from the Theorem of Artin that if $x$ is any element of an alternative algebra $A$ over $F$, and if $f(\lambda)$ is any polynomial in $\lambda$ with coefficients in $F$, then $f\left(R_{x}\right)=R_{f(x)}$ and $f\left(L_{x}\right)=L_{f(x)}$.

Moreover, if an alternative algebra $A$ contains a right nonsingular element $x$ and a left nonsingular element $y$, then $A$ has a unity quantity. For the identity transformation $I$ is then a polynomial in $R_{x}$ with coefficients in $F$, and the correspondence $x \rightarrow R_{x}$ is one-to-one. Thus the unity quantity of $A$ and the inverse $x^{-1}$ exist, and are polynomials in $x$, and $R_{x^{-1}}=\left(R_{x}\right)^{-1}$. Clearly these results hold for an alternative division algebra $A$.

In this paper we shall require the following lemma.

LEMMA 2. In an alternative division algebra $A$, the norm of a product $x y$ is equal to the product of the norms of $x$ and $y$.

For if $x=0$ the lemma is obvious. Otherwise $L_{x}$ is nonsingular, and $L_{x} R_{x} R_{y}=R_{x y} L_{x}$ by Lemma 1 . Thus $\left|L_{x}\right| \cdot\left|R_{x}\right| \cdot\left|R_{y}\right|=\left|R_{x y}\right| \cdot\left|L_{x}\right|$, and $\left|R_{x}\right| \cdot\left|R_{y}\right|=\left|R_{x y}\right|$. The conclusion follows.

Since any simple algebra $A$ over $F$ is central simple over its transformation center, the determination of all simple alternative algebras consists of a determination of those which are central simple. Zorn's results imply that a central simple alternative algebra over an arbitrary field is either (1) a division algebra, (2) an associative algebra, or (3) a Cayley-Dickson algebra with divisors of zero. ${ }^{6}$ We are led directly to this theorem.

ThEOREM 1. Let $A$ be an alternative, but not associative, central division algebra over $F$. Then $A$ is an algebra of degree two and order eight over $F$.

For there exists a scalar extension $A_{K}$ of $A$ such that $A_{K}$ over $K$ is not a division algebra. Since $A_{K}$ is not associative, $A_{K}$ is a CayleyDickson algebra (with divisors of zero) over $K$. Hence $A$ is of degree two and order eight over $F$.

2. Alternative division algebras of degree two. We are able to make a study of alternative division algebras of degree two which is independent of Zorn's results, and (although a portion of the result is indicated in Theorem 1) we shall do so. For the proof of Theorem 2 we require the two lemmas which follow.

${ }^{6}$ Zorn's so-called "vector matrix algebra" is (over any field, including those of characteristic two) merely a Cayley-Dickson algebra with divisors of zero. 
LEMMA 3. Let the principal function of any element $x$ of an alternative division algebra of degree two over $F$ be $x^{2}-t(x) \cdot x+n(x)$, where $t(x)$ and $n(x)$ are in $F$. Then the linear transformation

$$
S: \quad x \leftrightarrow x S=t(x)-x
$$

is an involution of $A$ such that $x+x S=t(x)$, and $x(x S)=(x S) x=n(x)$.

The conclusions are trivial except for showing that $S$ is an involution of $A$. Clearly $S^{2}=I$. By Lemma 2 we have $(x y) \cdot(x y) S=n(x y)$ $=n(x) \cdot n(y)=(x \cdot x S) \cdot n(y)$. It follows from the Theorem of Artin that $(x y) S=(x y)^{-1} \cdot(x y) \cdot(x y) S=y^{-1} x^{-1} x(x S) \cdot n(y)=n(y) \cdot y^{-1}(x S)$ $=(y S \cdot y) y^{-1}(x S)=y S \cdot x S$. Hence $S$ is an involution of $A$.

LEMMA 4. Let $B$ be an alternative algebra of order 2 sover $F$ defined as the supplementary sum $B=f_{1} D+\cdots+f_{s} D$, where $D=\left(1, u_{2}\right), u_{2}^{2}=u_{2}+\alpha$, $-4 \alpha \neq 1, f_{1}=1, f_{i}^{2}=\gamma_{i} \neq 0, \gamma_{i}$ in $F, d \cdot f_{i}=f_{i} \cdot d S$ for all $d$ in $D, S$ as in (1), $(i=2, \cdots, s)$. Then, if $B$ is a proper subalgebra of an alternative division algebra $A$ of degree two over $F$, there exists an element $g$ in $A$, but not in $B$, such that

$$
g^{2}=\gamma \neq 0, \gamma \text { in } F, \text { and } x g=g(x S)
$$

holds for all $x$ in $B$.

It is evident that $f_{j} D \cdot f_{j} D \subseteq D$ and that the intersection $\left(f_{j} D \cdot f_{k} D\right)$ $\cap D=0$ for $j \neq k, j, k=1,2, \cdots, s$. In order to establish (2) it is sufficient to prove the existence of $g$ with the trace $t(x g)=0$ for all $x$ of $B$. Now if $y$ is any element of $B$, then $y=d_{1}+f_{2} d_{2}+\cdots+f_{s} d_{s}$ with $d_{i}$ in $D$. But $t\left(f_{j} d_{i}\right)=0$ for $j \neq 1$. Hence $t(y)=t\left(d_{1}\right)$.

Let $v$ be an element of $A$ not in $B$, and write $g=\left(\lambda_{1}+\lambda_{2} u_{2}\right)$ $+f_{2}\left(\lambda_{3}+\lambda_{4} u_{2}\right)+\cdots+f_{s}\left(\lambda_{2 s-1}+\lambda_{2 s} u_{2}\right)+v$, where the $\lambda_{i}$ are undetermined coefficients in $F$. Denote $t\left(f_{j} v\right)$ by $\mu_{2 j-1}$ and $t\left(f_{j} u_{2} \cdot v\right)$ by $\mu_{2 j}$. Then the existence of $g$ satisfying (2) is equivalent to the existence of $\lambda_{i}(i=1, \cdots, 2 s)$ in $F$ such that

$$
\begin{array}{rlrlrl}
t(g) & =2 \lambda_{1}+ & \lambda_{2} & +\mu_{1} & =0, & \\
t\left(u_{2} g\right) & =\lambda_{1}+(1+2 \alpha) \lambda_{2} & +\mu_{2} & =0, \\
t\left(f_{j} g\right) & = & 2 \gamma_{j} \lambda_{2 j-1}+\gamma_{j} \lambda_{2 j}+\mu_{2 j-1} & =0, & j=2, \cdots, s, \\
t\left(f_{j} u_{2} \cdot g\right) & = & \gamma_{j} \lambda_{2 j-1}-2 \alpha \gamma_{j} \lambda_{2 j}+\mu_{2 j} & =0, & j=2, \cdots, s .
\end{array}
$$

But the determinant $\Delta$ of the coefficients of this system of linear equations is $\Delta=(1+4 \alpha) \cdot \prod_{2}^{s} \gamma_{j}^{2}(-1-4 \alpha) \neq 0$. Hence the desired solutions $\lambda_{i}$ exist.

An algebra $Q$ is called a quaternion algebra if $Q=\left(1, u_{2}, u_{3}, u_{4}\right)$, $u_{4}=u_{3} u_{2}, u_{2}^{2}=u_{2}+\alpha, u_{3}^{2}=\beta, u_{2} u_{3}=u_{3}\left(1-u_{2}\right)$, where $\alpha$ and $\beta \neq 0$ are in $F$, 
$-4 \alpha \neq 1$. Moreover, $Q$ is a division algebra if and only if there exist no $\lambda$ and $\mu$ in $F$ such that $\beta=\lambda^{2}+\lambda \mu-\alpha \mu^{2}$.

An algebra $C$ is called a Cayley-Dickson algebra if $C=Q+g Q$, with elements $z=x+g y$, where $x, y$ are quaternions, and multiplication is defined by

$$
\left(x_{1}+g y_{1}\right)\left(x_{2}+g y_{2}\right)=\left(x_{1} x_{2}+\gamma y_{2} \cdot y_{1} S\right)+g\left(x_{1} S \cdot y_{2}+x_{2} y_{1}\right),
$$

where $g^{2}=\gamma \neq 0$ in $F$ and $S$ is the involution (1) of $Q$.

TheOREM 2. An alternative algebra $A$ over $F$ is a division algebra of degree two over $F$ if and only if $A$ is one of the following:

(a) a separable quadratic field, or an inseparable field of exponent two,

(b) a quaternion division algebra $Q$, or

(c) a Cayley-Dickson algebra $C=Q+g Q$, where $Q$ is a division algebra and there exist no $\lambda, \mu, \rho, \sigma$ in $F$ such that

$$
\gamma=\lambda^{2}+\lambda \mu-\alpha \mu^{2}-\beta \rho^{2}-\beta \rho \sigma+\alpha \beta \sigma^{2} .
$$

If $A$ is generated by less than three elements, then $A$ is associative and is either (a) or (b). Otherwise, if the characteristic of $F$ is not two, $A$ contains a quaternion subalgebra $Q$ as in (b), and an element $v$ which is not in $Q$. If the characteristic of $F$ is two, consider two cases: if $A$ is commutative, then $A$ is associative ${ }^{7}$ and is either (a) or (b). If $A$ is not commutative, there exist two noncommutative elements $x, y$. These generate an associative, noncommutative subalgebra (a quaternion algebra $Q$ ) of $A$. Also there exists an element $v$ of $A$ which is not in $Q$.

The algebra $Q$ is a particular example, $s=2$, of the algebra $B$ defined in Lemma $4, f_{2}=u_{3}, \gamma_{2}=\beta$. Thus there exists an element $g$ in $A$, but not in $Q=B$, satisfying (2). Then $A$ contains $A_{0}=B+g B$, the elements $z$ of $A_{0}$ being expressible uniquely as $z=x+g y$ for $x, y$ in $B$. We make effective use of equation (2), Lemma 1 , and the Theorem of Artin in proving that $A_{0}$ is an algebra in which multiplication is defined by (3).

For $\left(g y_{1}\right)\left(g y_{2}\right)=\left(g y_{1}\right)\left(y_{2} S \cdot g\right)=\left[g\left(y_{1} \cdot y_{2} S\right)\right] g=\left[\left(y_{2} \cdot y_{1} S\right) g\right] g=\left(y_{2} \cdot y_{1} S\right) g^{2}$ $=\gamma y_{2} \cdot y_{1} S$. Also from the fact that $y_{1} S=\gamma^{-1} y_{1} S \gamma=\gamma^{-1} y_{1} S \cdot g^{2}$ $=\gamma^{-1}\left(y_{1} S \cdot g\right) g=\gamma^{-1}\left(g y_{1}\right) g$, it follows that $g\left(x_{2} y_{1}\right)=\left(y_{1} S \cdot x_{2} S\right) g$ $=\left[\left\{\gamma^{-1}\left(g y_{1}\right) g\right\} x_{2} S\right] g=\gamma^{-1}\left(g y_{1}\right)\left[g\left(x_{2} S \cdot g\right)\right]=\gamma^{-1}\left(g y_{1}\right)\left[g\left(g x_{2}\right)\right]=\gamma^{-1}\left(g y_{1}\right)$ $\cdot\left(\gamma x_{2}\right)=\left(g y_{1}\right) x_{2}$. But then $x_{1}=\gamma^{-1}\left(g \cdot x_{1} S\right) g$ and $g\left(y_{2} S \cdot x_{1}\right)=\left(g x_{1}\right) \cdot y_{2} S$ also. Hence $x_{1}\left(g y_{2}\right)=\left[\gamma^{-1}\left(g \cdot x_{1} S\right) g\right]\left(g y_{2}\right)=\gamma^{-1}\left[g\left(x_{1} S \cdot g\right)\right]\left(y_{2} S \cdot g\right)$ $=\gamma^{-1}\left[g\left\{\left(x_{1} S \cdot g\right) y_{2} S\right\}\right] g=\gamma^{-1}\left[g\left\{\left(g x_{1}\right) y_{2} S\right\}\right] g=\gamma^{-1}\left[g\left\{g\left(y_{2} S \cdot x_{1}\right)\right\}\right] g$ $=\gamma^{-1}\left(\gamma y_{2} S \cdot x_{1}\right) g=g\left(x_{1} S \cdot y_{2}\right)$. Hence equation (3) holds.

${ }^{7}$ A commutative alternative algebra over a field of characteristic not three is associative. See M. Zorn, Theorie der Alternativen Ringe, op. cit. 
Having shown that any alternative division algebra of degree two, which is not associative, must contain the algebra $A_{0}$, we need now only to prove that $A_{0}$ is actually an alternative algebra and is not a proper subalgebra of any alternative division algebra of degree two. We may readily verify these conclusions if we refer to the matrices corresponding to the linear transformations we have used. Thus, for $z=x+g y$ in $A_{0}$,

$$
\begin{aligned}
R_{z} & =\left(\begin{array}{cc}
R_{x} & S R_{y} \\
\gamma S L_{y} & L_{x}
\end{array}\right), \\
L_{z} & =\left(\begin{array}{cc}
L_{x} & R_{y} \\
\gamma R_{y S} & L_{S x}
\end{array}\right)
\end{aligned}
$$

where the matrices $R_{x}, L_{x}, \cdots, S$ are the 4-by-4 matrices of the linear transformations induced on $Q$ by the corresponding transformations on $A_{0}$. Computation, involving the associativity of $Q$, yields $R_{z^{2}}=\left(R_{z}\right)^{2}$ and $L_{z^{2}}=\left(L_{z}\right)^{2}$. Hence the Cayley-Dickson algebra $C=A_{0}$ is alternative. If $C$ were a proper subalgebra of $A$, then $A$ would contain a new $A_{0}=C+h C$ with multiplication defined by (5), $x$ and $y$ being in $C$. But computation with the matrices above reveals that if $A_{0}$ were alternative, $C$ would be associative, which it is not.

Condition (4) follows from Lemmas 2 and 3. Let $z=x+g y$ with $x, y$ in $Q$, and let $S$ be defined by (1). Then $n(z)=z \cdot z S=(x+g y)(x S+y S \cdot g S)$ $=(x+g y)(x S-g y)=x \cdot x S-\gamma y \cdot y S=n(x)-\gamma n(y)$. Now $C$ is a division algebra if and only if $n(z) \neq 0$ for every nonzero $z$ in $C$. But $n(x)-\gamma n(y)=0$ if and only if $\gamma=n(x)[n(y)]^{-1}=n\left(x y^{-1}\right)=n(v)$, the norm of $v$ for some $v$ in $Q$. Let $v=\lambda+\mu u_{2}+\rho u_{3}+\sigma u_{4}$. Then $C$ is a division algebra if and only if there exist no $\lambda, \mu, \rho, \sigma$ in $F$ such that $\gamma=n(v)=v \cdot v S=\lambda^{2}+\lambda \mu-\alpha \mu^{2}-\beta \rho^{2}-\beta \rho \sigma+\alpha \beta \sigma^{2}$.

We may combine the results of Zorn and Theorems 1 and 2 in the following manner and say: an alternative, but not associative, algebra $A$ over an arbitrary field $F$ is central simple if and only if $A$ is a Cayley-Dickson algebra over $F$.

3. Isotopes of alternative algebras. Albert has proved that an algebra $A$ with a unity quantity is associative if and only if every isotope of $A$ with a unity quantity is associative and is equivalent to $A$. We consider here the corresponding problem for alternative algebras.

ThEOREM 3. Let $A$ be an alternative algebra, and $B$ be an isotope of $A$ with a unity quantity. Then $A$ has a unity quantity, and $B$ is alternative. 
For $B$ is equivalent to a principal isotope $A_{0}$ of $A$, in which products $(a, x)$ are defined by $(a, x)=a R_{x}^{(0)}$ with $R_{x}^{(0)}=P R_{x Q}$ for nonsingular transformations $P, Q$. (In terms of left multiplications, $(x, a)=a L_{x}^{(0)}$ with $L_{x}^{(0)}=Q L_{x P}$.) Let $e$ be the unity quantity of $A_{0}$ and $h=e Q$. Then $I=R_{e}^{(0)}=P R_{h}$, and $R_{h}$ is nonsingular. Similarly if $k=e P$, then $L_{k}$ is nonsingular, and it follows from the proof in $\$ 1$ that the algebra $A$ has a unity quantity.

Now $P=R_{h}^{-1}=R_{h^{-1}}$. Hence $R_{x}^{(0)}=R_{h^{-1}} R_{x Q}=L_{h} R_{h^{-1}(x Q)} L_{h^{-1}}$ by Lemma 1. That is, $R_{x}^{(0)}=H R_{(x T) H} H^{-1}$ where $H=L_{h}$ and $T=Q L_{h}^{-2}$. Hence $A_{0}$ is equivalent to an isotope $A_{1}$ of $A$ in which products are denoted by $[a, x]=a R_{x}^{(1)}$ where $R_{x}^{(1)}=R_{x T}$. Also $[x, a]=a L_{x}^{(1)}$ where $L_{x}^{(1)}=$ $T L_{x}$. Let $f$ be the unity quantity of $A_{1}$. Then $I=L_{f}^{(1)}=T L_{f}$ and $T=L_{f}^{-1}=L_{f^{-1}}$. Hence $R_{x}^{(1)}=R_{f^{-1} x}$. Therefore $R_{[x, x]}^{(1)}=R_{x R_{x}^{(1)}}^{(1)}=R_{x R_{f}{ }^{-1} x}$ $=R_{f^{-1}\left\{x\left(f^{-1} x\right)\right\}}=R_{\left(f^{-1} x\right)^{2}}=R_{f^{-1} x} R_{f^{-1} x}=R_{x}^{(1)} R_{x}^{(1)}$ since $A$ is alternative. Since $B$ is equivalent to $A_{1}$, it follows that half of the alternative law holds in $B$.

But similarly $B$ is equivalent to an isotope $A_{2}$ of $A$ in which products are denoted by $\{x, a\}=a L_{x}^{(2)}$ where $L_{x}^{(2)}=L_{x c^{-1}}$, the element $c$ being the unity quantity of $A_{2}$. Then $L_{\{x, x\}}^{(2)}=L_{x}^{(2)} L_{x}^{(2)}$, and the second half of the alternative law holds in $B$.

We complete the study of isotopy for simple alternative algebras by proving the following theorem.

TheOREm 4. Let $A$ be a Cayley-Dickson algebra, and $B$ be any isotope of $A$ with a unity quantity. Then $B$ is equivalent to $A$.

Any isotope with a unity quantity of a central simple algebra $A$ with a unity quantity is also central simple. Therefore, any isotope $B$ with a unity quantity of a Cayley-Dickson algebra $A$ is also a CayleyDickson algebra. We shall show that $B$ is equivalent to $A$.

For $B$ is equivalent to an algebra $A_{1}$ in which products are denoted by $[a, x]=a R_{x}^{(1)}$ where $R_{x}^{(1)}=R_{f^{-1} x}$, the element $f$ being the unity quantity of $A_{1}$. Now $f$, as an element in $A$, is contained in some quaternion subalgebra $Q$ of $A$. Let $x$ range over $Q$, and $R$ be the subspace of $A_{1}$ consisting of all elements $f x$. Then $x \leftrightarrow f x$ is an equivalence of $Q$ and $R$. For since $Q$ contains $f$ and is associative, $[f x, f y]=f x R_{f-1} f y$ $=f(x y)$ for all $x, y$ in $Q$.

Let $S$ be the involution of $Q$ defined by (1), and let $z=f x$. Then the transformation

$$
U: \quad z \leftrightarrow z U=f(x S)
$$

is the corresponding involution of $R$. Now $A=Q+g Q$ as in (3). Also $A_{1}=R+w R$, where $w=f g$ (and where the multiplication defining $w R$ 
is of course the multiplication in $A_{1}$ ). By the proof of Theorem 2 , in order to show the equivalence of $A$ and $A_{1}$ it is sufficient to show that $[w, w]=\gamma f$ and $[w, z]=[z U, w]$ for every $z$ of $R$. But $[w, w]$ $=w\left(f^{-1} w\right)=(f g)\left(f^{-1} f g\right)=f g^{2}=\gamma f$, and $[w, z]=w\left(f^{-1} z\right)=(f g)\left(f^{-1} f x\right)$ $=(f g) x=g(x \cdot f S)=(f \cdot x S) g=(f \cdot x S)\left(f^{-1} f g\right)=z U\left(f^{-1} w\right)=[z U, w]$. This proves the theorem.

Newport News, Va.

\section{ON FIBRE SPACES. I}

RALPH H. FOX

In subsequent papers I propose to investigate various properties of fibre spaces. ${ }^{1}$ The object of the fundamental Hurewicz-Steenrod definition ${ }^{1}$ is to state a minimum ${ }^{2}$ set of readily verifiable conditions under which the covering homotopy theorem ${ }^{1}$ holds. An apparent defect of their definition is that it is not topologically invariant. In fact, for topological space $X$ and metrizable non-compact space $B$ the property " $X$ is a fibre space over $B$ " depends on the metric of $B$. The object of this note is to give a topologically invariant definition of fibre space and to show that (when $B$ is metrizable) $X$ is a fibre space over $B$ in this sense if and only if $B$ has a metric in which $X$ is a fibre space over $B$ in the sense of Hurewicz-Steenrod. Since the definition of fibre space is controlled by the covering homotopy theorem, an essential part of my program is to give a topologically invariant definition of uniform homotopy.

Let $\pi$ be a continuous mapping of a topological space $X$ into another topological space $B$. Let $\Delta=\Delta(B)$ denote the diagonal set $\sum_{b \in B}(b, b)$ of the product space $B \times B$ and let $\bar{\pi}$ denote the mapping of $X \times B$ into $B \times B$ which is induced by the mapping $\pi$ according to the rule $\bar{\pi}(x, b)=(\pi(x), b)$. Thus the graph $G$ of $\pi$ is the set $\bar{\pi}^{-1}(\Delta)$, and $\bar{\pi}^{-1}(U)$ is a neighborhood of $G$ whenever $U$ is a neighborhood of $\Delta$.

Any neighborhood $U$ of $\Delta$ determines uniquely a covering of $B$ by neighborhoods $N_{U}(b)$ according to the rule $b^{\prime} \in N_{U}(b)$ when $\left(b, b^{\prime}\right) \in U$.

Received by the editors January 13, 1943.

${ }^{1}$ W. Hurewicz and N. E. Steenrod, Proc. Nat. Acad. Sci. U.S.A. vol. 27 (1941) p. 61 .

${ }^{2}$ How well they succeeded in this will be indicated in my next communication. 